\title{
Joint Link-level and Network-level Reconfiguration for mmWave Backhaul Survivability in Urban Environments
}

\author{
Yuchen Liu, Qiang Hu and Douglas M. Blough \\ Georgia Institute of Technology, Atlanta, GA, 30332
}

\begin{abstract}
mmWave communication has been recognized as a highly promising technology for $5 \mathrm{G}$ wireless backhaul, which is capable of providing multi-gigabit per second transmission rates. However, in urban wireless backhaul environments, unforeseen events can cause short-term blockages or node failures and, therefore, network survivability is extremely important. In this paper, we investigate a novel relay-assisted mmWave backhaul network architecture, where a number of small-cell BSs and relays are deployed, e.g. on the lampposts of urban streets. Relays are used to provide multihop line-of-sight paths between small-cell BSs, which form logical links of the network. In this scenario, the interconnected logical links make up a mesh network, which offers opportunities for both link-level and network-level reconfiguration. We propose two joint link-network level reconfiguration schemes for recovery after exceptional events. One prioritizes relay path (link-level) reconfiguration and uses alternate network-level paths only if necessary. The other splits traffic on both reconfigured links and backup paths to improve network throughput. Simulation results demonstrate that the proposed schemes significantly outperform purely link-level and purely network-level reconfiguration schemes. The proposed approaches are shown to not only maintain high network throughput but to also provide robust blockage/fault tolerance across a range of scenarios for urban mmWave backhaul networks.
\end{abstract}

\section{CCS CONCEPTS}

- Networks $\rightarrow$ Network reliability; Network protocol design; Network performance analysis.

\section{KEYWORDS}

Millimeter wave; wireless backhaul; relays; survivability; reconfiguration; robustness

\section{ACM Reference Format:}

Yuchen Liu, Qiang Hu and Douglas M. Blough. 2019. Joint Link-level and Network-level Reconfiguration for mmWave Backhaul Survivability in Urban Environments. In 22nd Int'l ACM Conference on Modeling, Analysis and Simulation of Wireless and Mobile Systems (MSWiM '19), November 25-29, 2019, Miami Beach, FL, USA. ACM, New York, NY, USA, 9 pages. https://doi.org/10.1145/3345768.3355913

Permission to make digital or hard copies of all or part of this work for personal or classroom use is granted without fee provided that copies are not made or distributed for profit or commercial advantage and that copies bear this notice and the full citation on the first page. Copyrights for components of this work owned by others than ACM must be honored. Abstracting with credit is permitted. To copy otherwise, or republish, to post on servers or to redistribute to lists, requires prior specific permission and/or a fee. Request permissions from permissions@acm.org.

MSWiM '19, November 25-29, 2019, Miami Beach, FL, USA

(c) 2019 Association for Computing Machinery.

ACM ISBN 978-1-4503-6904-6/19/11 . \$ \$15.00

https://doi.org/10.1145/3345768.3355913

\section{INTRODUCTION}

With the advent of $5 \mathrm{G}$ networks, it is expected that a large number of small-cell base stations (BSs) will be deployed in urban areas. Each small cell will cover a smaller area but will have much higher capacity and less interference. Millimeter wave (mmWave) communication is very well suited to this scenario, especially for self-backhauling [1-3], wherein a huge amount of data must be transmitted with high rates among the BSs.

Due to the trend for dense deployment that makes current wired backhaul costly and in some cases prohibitive, wireless backhaul becomes a very promising solution [4], [5]. With wireless backhaul, only a very small portion of BSs, referred to as anchored BSs (A-BS), will have fiber backhaul and the rest of the small-cell BSs (SBSs) backhaul to the fiber sites wirelessly. In order to fully realize mmWave wireless backhaul in urban environments, the poor propagation characteristics and higher penetration loss of mmWave communications are two challenges that must be overcome. With high-gain directional antennas and relaying techniques, a sequence of relatively short but very high rate mmWave links can be produced to compensate the high path loss.

Severe penetration loss makes blockage effects a serious problem in mmWave networks $[6,7]$, because the signal strength is degraded by about $30 \mathrm{~dB}$ for non-line-of-sight (NLOS) paths when mmWave links are blocked by obstacles such as buildings, vehicles, or even humans [8]. For this reason, it is necessary to adopt an appropriate network topology with effective path reconfiguration mechanisms, which can be applied for maintenance of line-of-sight (LOS) mmWave paths with high rates in the presence of blockages.

There are primarily two kinds of network architecture for mmWave backhaul, which are the centralized and distributed architectures [9]. In a centralized architecture, a macrocell BS (M-BS) is situated in the center with a number of SBSs distributed around it, and these SBSs do not communicate with each other by direct links but rather they access the core network through the M-BS that is connected to the gateway by fiber links. This kind of system forms a star topology, which makes it hard to perform path reconfiguration for potential link failures since there exists only one route from each SBS to the M-BS. However, in a distributed architecture, backhaul data of each SBS are relayed to an A-BS instead of the M-BS, and these data are allowed to traverse mmWave links of adjacent SBSs and finally are collected by the A-BS to the core network through fiber links [10]. Therefore, a mesh-like backhaul network will be formed by the mmWave links. Compared with the centralized solution, the distributed architecture can achieve higher throughputs and be more reliable, mainly owing to sharing cooperative traffic among multiple wireless SBSs. In this architecture, when the communication of a path is blocked, the data can be transmitted to other adjacent SBSs and be routed on an alternate path to maintain the high-rate connectivity. 
In this paper, we consider a relay-assisted mmWave backhaul network architecture in urban environments, where a number of SBSs and mmWave dedicated relays are deployed along the urban streets, which naturally produces a mesh network structure. The deployment of relays between each SBS pair provide multi-hop LOS relay paths between SBSs, which we refer to as logical links in the mesh network. These networks are susceptible to node failures ${ }^{1}$ and/or obstacles in the form of large trucks or other objects that could block some of the primary LOS paths. An advantage of the use of multi-hop logical links is that they allow for link-level reconfiguration when an obstacle blocks an individual physical link. This provides opportunities for both link-level and network-level reconfiguration for network survivability in the presence of obstacles and/or node failures.

We propose two joint link-network level reconfiguration schemes to tolerate these exceptional events. The first approach is to prioritize link-level reconfiguration, and use a network-level backup path only when the performance of the reconfigured logical link drops below a threshold value. The second approach is designed to further improve the throughput performance, by using both reconfigured logical links and alternative backup paths for data transmission when blockage occurs. In this case, the traffic is split optimally and off-loaded to these multiple paths. Through simulation, we evaluate the performance of our proposed algorithms on backhaul networks under random obstacle scenarios. The results show that our proposed schemes substantially outperform purely link-level and purely network-level reconfiguration approaches. The results also demonstrate that our proposed approaches can provide nearoptimal blockage tolerance while maintaining throughputs that can satisfy the rigorous data demands of wireless backhaul scenarios.

\section{RELATED WORK}

Prior work on path recovery in wireless networks can be broadly categorized into two main methods: network-level reconfiguration (NLR) and link-level reconfiguration (LLR).

\subsection{Network-level reconfiguration schemes}

In general, network-level reconfiguration includes the protection method and the restoration method [12]. The former method reserves backup resources before link failure occurs, whereas the restoration method finds a new path for the influenced traffic after failure occurs. Most related works focus on the protection method to prevent the link failure since it can achieve fast recovery [13-15]. The main idea in these approaches is to select a single disjoint backup path for each primary path based on the assumption that it is unlikely for a primary path and its backup path to fail simultaneously. [16] proposed a dynamic routing algorithm to select the best backup path with minimized total cost, which improves the utilization and reduces blocking in mesh networks. [17] introduced another method that is based on pre-finding a set of backup paths for each active path, and multiple survivable paths of a pair of nodes could be used to survive any single risk. In [18], the author

\footnotetext{
${ }^{1}$ The node-failure case is actually equivalent to a blockage case where an obstacle totally blocks the node. Thus, in the remainder of the paper, we mainly focus on blockage effects caused by random obstacles.
}

presented a predictive weather-assisted routing protocol that routes data around potential link failures ahead of time.

This kind of reconfiguration approach can also be applied in backhaul networks, where the central A-BS pre-computes several eligible backup paths for primary working paths, and once the blockage occurs between a pair of SBSs, the A-BS can efficiently switch to an alternate backup path as the new network-level path to maintain the high-rate backhaul data transmission. However, this approach is topology-dependent, and can only be applied in a mesh-like network, because if the network has a tree or star-like topology, there exists only one route between the A-BS any given SBS, and it is impossible to find any other network-level paths for blockage avoidance. In addition, backup paths might not always exist [17], because sometimes it is hard to find a candidate path that satisfies both rate and path length requirements of mmWave backhaul.

\subsection{Link-level reconfiguration schemes}

Another approach to blockage handling is based on link-level reconfiguration (LLR), which aims to reconfigure a new relay path within a single logical link between two wireless nodes when a physical link failure occurs. To the best of our knowledge, there are few works that considered multi-hop relay paths for blockage avoidance in outdoor environments [19], [20], and they are primarily concerned with finding a relay path with the highest probability of reaching the BS. In contrast, our work considers the maintenance of backhaul paths with very high rates in the presence of temporary blockages. In our previous work [21], the high-throughput pathlevel reconfiguration (HTPR) algorithm is proposed to reconfigure around temporary blockages in relay-assisted mmWave backhaul networks. In most situations, the HTPR scheme maintains highrate connectivity for logical links between each SBS pair and, in this work, we use it as our comparison point for pure link-level reconfiguration schemes.

Here we note that LLR can be applied in all types of network topologies with relay assistance, and it can be conducted at the SBS level without the participation of the A-BS. However, the link quality will be degraded after reconfiguration since alternative physical links are always longer than the original link. On the other hand, while LLR may provide some network control benefits, it cannot handle some blockage cases where the logical link (relay path) is totally blocked.

\section{SYSTEM OVERVIEW}

In this section, we introduce the network topology, and channel and antenna models used in the remainder of the paper.

\subsection{Network model in an urban area}

For future $5 \mathrm{G}$ cellular networks, a large number of small-cell base stations will be deployed in urban areas to cooperatively provide a more reliable access experience for users. A number of works have proposed the use of mmWave wireless backhaul for $5 \mathrm{G}$ networks [22-24]. Here we consider a relay-assisted mmWave mesh network architecture for backhaul in urban areas [25], [26], where mmWave relay nodes are used to assist in connecting BSs of the wireless mesh network. 


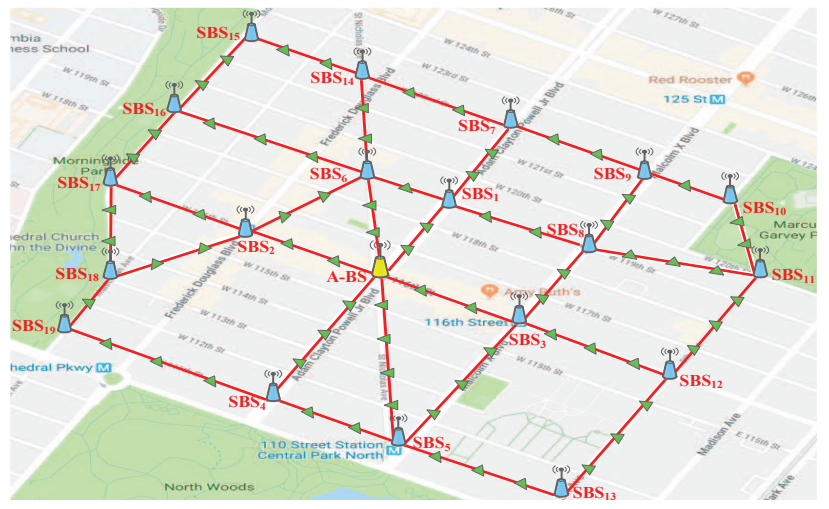

Figure 1: mmWave backhaul network model in a section of Manhattan.

An example relay-assisted mesh backhaul network is shown in Fig. 1. In this example, there are a single A-BS connected with a fiber to the core network, a number of SBSs deployed at street corners, and several mmWave relays along the roadside (shown with the green triangles). It is assumed that all these three entities can communicate in the mmWave band using directional antennas with steerable beams, which produce a number of interconnected mmWave links to form a wireless mesh network. There are three important entities in our mmWave backhaul network model:

- Physical link: An actual link between relay pairs or between a relay and its adjacent BS - the capacity of physical links is determined by Shannon's Theorem.

- Logical Link (relay path): A path between BS pairs including SBS-to-SBS and A-BS-to-SBS, which consists of multiple physical links. The achievable capacity of each logical link is determined by the capacity of its bottleneck physical link pair [3]

- Backhaul path: A path between the A-BS and a SBS, which can consist of several logical links. For simplicity, we use $P_{0, i}$ to denote the backhaul path from the A-BS to $\mathrm{SBS}_{i}$.

In this work, we adopt the "street canyon" model for relay paths forming logical links [25]. In the street canyon model, each logical link between BSs runs along a street and consists of a sequence of mmWave relays. As in [21], we assume that relays are deployed in a regular fashion on both sides of the road, e.g. by deploying them on equally-spaced lampposts. As shown in Fig. 2, these physical links within a given logical link form a triangular-wave topology, where the topology angle $\theta$ and horizontal distance between adjacent nodes $d_{0}$ are the same everywhere along the topology (as depicted by the blue links of Fig. 2). One advantage of this topology is that the mutual interference along the logical link can be eliminated if $\theta$ is made large enough relative to the beamwidth $\phi$ of the directional antennas (Theorem 1 in [11]), i.e. if $\theta-\arctan \left(\frac{\tan \theta}{3}\right)>\frac{\phi}{2}$. In this way, the logical link can support the high throughput of $10+$ Gbps to meet the requirement of mmWave backhaul. On the other hand, this kind of topology is also capable of reconfiguring mmWave links to avoid obstacles (eg. parked large vehicles) that occur along the roadway. Through adaptive beam steering and dilation when one or more original links are blocked, alternative links can be used to restore the LOS connectivity of a logical link [27].

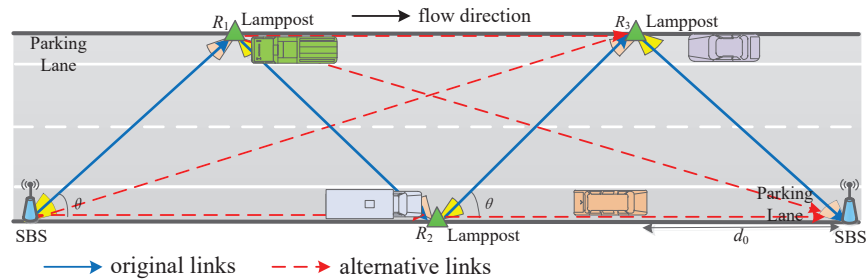

Figure 2: Original and alternative links in the triangularwave topology.

Note that the mmWave relay devices used in the network model are dedicated to a single logical link between a pair of BSs, because we assume relays are simple devices that cannot support the sharing between different logical links. In addition, we assume this kind of simple relay is subject to the primary interference constraint, which means a single relay cannot transmit and receive simultaneously. However, we assume that BSs (A-BS or SBSs) are not affected by these constraints since they are more complex devices with capability to use better antenna isolation and interference cancellation technologies.

From the view of the entire network model, the separation distances between each pair of BSs are around $350 \mathrm{~m}$, so the total modeled area in Fig. 1 is around $1200 \mathrm{~m} \times 1300 \mathrm{~m}$, which is a bit larger than the size of a typical $4 \mathrm{G}$ macrocell. By leveraging the geographical advantage with relaying technique in urban streets, this network architecture can not only eliminate mutual interference along logical links to achieve higher throughputs for backhaul, but it also naturally forms a mesh-like topology in an urban area, which makes it possible to jointly conduct the network-level and link-level reconfiguration for the improvement of network survivability.

\subsection{Channel and antenna model}

Here we make the standard assumption of additive white Gaussian noise channels. The rate of the directional unblocked physical link $p$ follows Shannon's Theorem with an upper limitation, i.e.,

$$
R_{p} \leq \beta \cdot B \cdot \log _{2}\left(1+\min \left\{\frac{P_{r}(d)}{N_{T}}, T_{\max }\right\}\right)
$$

where $B$ is channel bandwidth, $N_{T}$ is the power of thermal noise, $T_{\max }$ is the upper bound of operating signal-noise ratio due to the limiting factors like linearity in the radio frequency front-end, and the link utility ratio $\beta \in(0,1)$. Considering the primary interference of our simplified relays, $\beta \leq 0.5$, and a maximum end-to-end throughput of nearly 16 Gbps can theoretically be achieved in mmWave communications [3]. Here $P_{r}(d)$ is the received power of the intended transmitter's signal, and equals $k_{0} P_{t} G_{t} G_{r} d^{-\alpha}$, where $k_{0} \propto\left(\lambda_{w} / 4 \pi\right)^{2}, \lambda_{w}$ is the signal's wavelength, $d$ is the propagation distance, $\alpha$ is the path-loss exponent, and $G_{t}$ and $G_{r}$ are antenna gains at the transmitter and receiver, respectively.

In this work, a flat-top directional antenna model is adopted for each wireless node, which means that transceiver antennas have a high constant gain $G_{h}$ within the beam, and a very low gain $G_{l}$ that can be ignored outside the narrow beamwidth $\phi$. For example, with the 61 element uniform hexagonal array antenna simulated by MATLAB software, the antenna gains $G_{h}$ can be generated as 
$23.18 \mathrm{dBi}$ and $G_{l}$ is lower than $0 \mathrm{dBi}$ when the antenna beamwidth is around $15^{\circ}$.

\section{JOINT LINK-LEVEL AND NETWORK-LEVEL RECONFIGURATION SCHEME}

The ability to reconfigure logical links (relay paths) in the presence of obstacles provides multiple possible approaches to blockage tolerance for the mmWave backhaul network. Link-level blockage tolerance mainly adopts relay path reconfiguration schemes, i.e., finding an alternative physical link to substitute the original blocked link. As for network-level reconfiguration, it usually uses a new high-rate path between the source and destination nodes for blockage avoidance. In this section, we present joint link-network level reconfiguration schemes for fast recovery, which utilize relay path reconfiguration and alternate network-level paths in combination.

\subsection{Prioritized Link-level Reconfiguration}

In our considered relay-assisted network scenario, we first propose a prioritized link-level reconfiguration scheme (PLLR), which takes advantage of both link-level and network-level tolerance ability. The main idea is to prioritize relay path reconfiguration to avoid the use of alternate network-level paths whenever possible, so that the A-BS does not need to modify the routing table and inform the SBSs, which might complicate the network control. However, alternative network-level paths can be used if the performance of a reconfigured relay path drops so much that the throughput of that logical link is lower than a threshold value, which means that the original backhaul path $P_{0, i}$ cannot satisfy the data demand at $\mathrm{SBS}_{i}$. In what follows, we introduce this novel approach in detail.

\section{1) Backup path selection}

To achieve fast recovery, we pre-calculate a set of backup paths for each primary backhaul path and store them in the survivablepath set (SS). Different from other related works that just find one totally disjoint backup path to recover from a single link failure, the SS is used to overcome multiple blockages since obstacles could block a primary path and some of its backup paths simultaneously in our considered scenario. On the other hand, there may not always exist a totally disjoint backup path which also satisfies the high-rate and few-hop constraints, but some partially disjoint paths that meet the requirements can avoid the blockage and could even have better performance than a totally disjoint backup path.

Therefore, our approach finds a SS that consists of a set of backup paths that may not be totally disjoint but can survive blockages, and this enables us to provide protection for a broader range of scenarios and increase the network survivability. Here the backup path selected into SS for potential path recovery should satisfy two requirements: 1) supporting high throughput for mmWave backhaul; 2) with fewer hops than a specified threshold, because too many hops will lead to an unacceptably high end-to-end latency between the A-BS and the associated SBS.

The approach of finding backup paths with higher rates and hop-count constraint is shown in Algorithm 1. Considering the hop counts of different logical links in the topology, we first find the minimum hop counts $m$ among these logical links. Then, given a

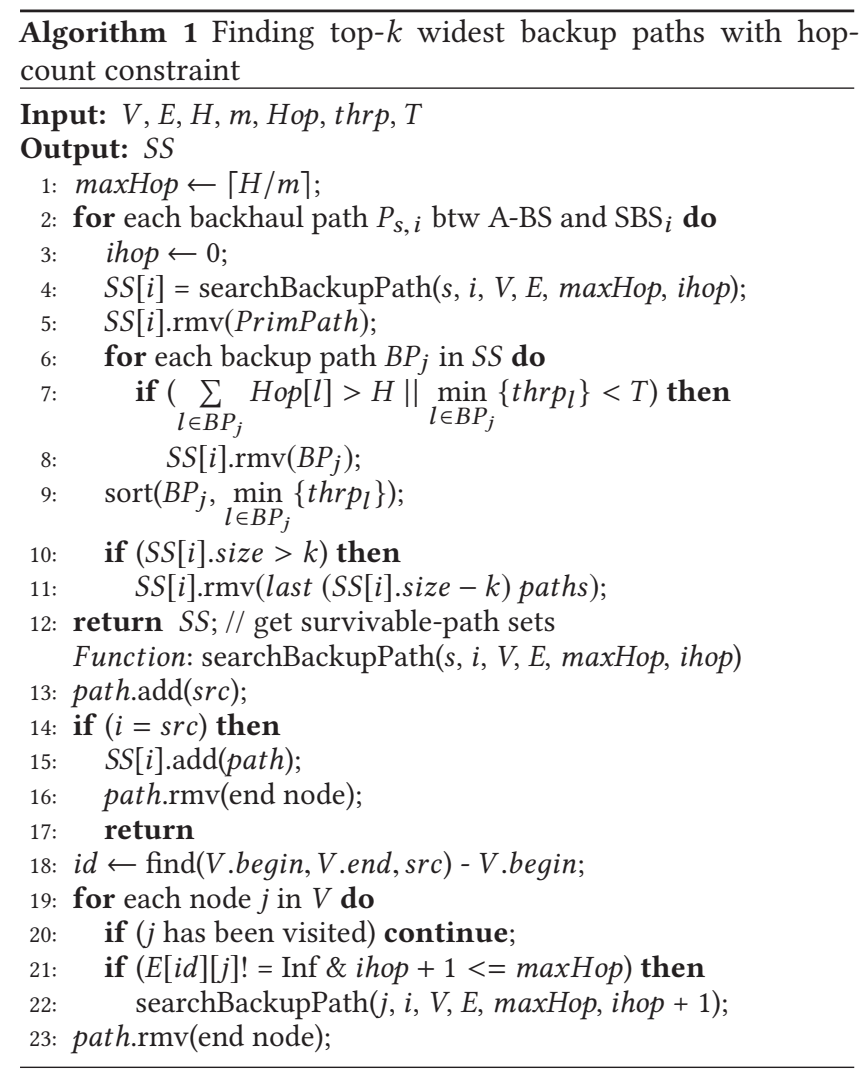

hop-count constraint $H$ (including intermediate relays and relayedSBSs), we compute a coarse allowable logical-level hops as the rounded-up $H / m$ (Line 1). After conducting the depth-first search (DFS), we find all backup paths which have their logical-level hop counts ihop no more than maxHop (Line 13-23), and these paths can be further put into survivable path set $S S[i]$ only if they satisfy the following two conditions: 1 ) the total hop counts of this path are not larger than $H$, because maxHop used in DFS steps actually relaxes the real hop-count constraint, and some searched backup paths with more than $H$ hops need to be dropped; 2) the throughput of bottleneck logical link pair within this backup path should meet the high-rate backhaul requirement $T$ (Lines 7-8).

Note that with an appropriate $H$ in most network topologies, there are only a small number of backup paths in each SS. However, we also set an upper bound $k$ on the number of backup paths in case the path length constraint is so weak that a large number of possible backup paths exist. In this scenario, we only put the $k$ backup paths with highest rates into SS (Lines 10-12).

\section{2) Combined link-level and network-level reconfiguration}

Algorithm 2 shows the pseudocode for the proposed PLLR scheme, which jointly considers link-level and network-level reconfigurations. In the "Input" arguments, $C$ is a vector that includes the achievable capacity of each logical link between BSs, and NP is a path set that contains each backhaul path $P_{0, k}$ between A-BS and $\mathrm{SBS}_{k}$ in the network. $D$ and $A D$ are the sets that include data-rate demand of each SBSs and the aggregated data-rate demand of each logical link, respectively, and the link much closer to the A-BS will 
have higher aggregated data-rate demand since it is required to carry more traffic to further SBSs. SS contains the survivable-path set $S S_{k}$ of each $P_{0, k}$. When the logical link $\mathcal{L}$ between $\mathrm{BS}_{i}$ and $\mathrm{BS}_{j}$ is blocked, the high throughput link-level reconfiguration algorithm (HTPR) [21] is first executed for logical link recovery, and the achievable capacity of $\mathcal{L}$ will be degraded since one or more longer physical links would be selected for blockage avoidance, and then $C$ is updated with the new capacity of $\mathcal{L}$ (Lines 1-3).

If the current throughput of the degraded link $\mathcal{L}$ drops lower than its aggregated data-rate demand (AD) (Line 3), all SBSs backhauled by the link $\mathcal{L}$ are found and put into $B_{\mathcal{L}}$ (Lines $4-5$ ). Then the network-level reconfiguration will be activated to select alternate backup paths for some of affected SBSs.

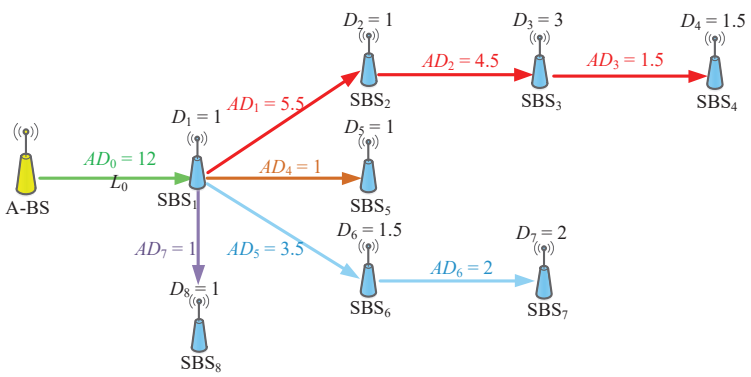

Figure 3: An example for minimizing rSBSs.

For example, Fig. 3 shows several primary backhaul paths, and every logical link has the achievable capacity of $12 \mathrm{Gbps}$ and datarate demand at each $\mathrm{SBS}_{i}$ is $D_{i}$. From further SBSs to A-BS, we can compute the $\mathrm{AD}$ of each logical link in turn (shown in Fig. 3). Now assuming the current capacity of link $\mathcal{L}_{0}$ (between $A B S$ and $\left.\mathrm{SBS}_{1}\right)$ drops to $C_{\mathcal{L}_{0}}\left(C_{\mathcal{L}_{0}}<A D_{0}\right)$, all backhauled SBSs $\left(\mathrm{SBS}_{1} \sim\right.$ $\mathrm{SBS}_{8}$ ) are obtained. In order to avoid the use of more alternate network-level paths which might complicate the network control, we set an objective to minimize the number of SBSs that need to be reconfigured to use backup paths (referred to as rSBSs).

One direct method of minimizing rSBSs (MRS) is to formulate the following optimization problem:

$$
\begin{gathered}
\max _{S} \sum_{i \in B_{l}} s_{i} \\
\text { s.t. } \sum_{i \in B_{l}} D_{i} \cdot s_{i} \leq C_{\mathcal{L}}, s_{i} \in\{0,1\}
\end{gathered}
$$

where $s_{i}$ is the SBS backhauled by the logical link $\mathcal{L}$. The objective function in Eq. (2) aims to satisfy greatest number of SBSs affected by this link. Eq. (3) indicates the capacity constraint, where the aggregated data-rate demand on the link $\mathcal{L}$ cannot exceed its current capacity, and $s_{i}$ will be set as 1 if $\mathrm{SBS}_{i}$ is chosen, otherwise it equals to 0 . This optimization problem is similar to the 0/1 knapsack problem, and in this case the optimal solution is to sort the SBSs in order of increasing data-rate demand and satisfy them in turn until there is not enough capacity of link $\mathcal{L}$ to backhaul any SBSs. As an example, in Fig. 3, when the capacity of the logical link between A-BS and $\mathrm{SBS}_{1}$ (shown with green line) drops to 7 , we need to reconfigure new bachaul paths for $\mathrm{SBS}_{3}$ and $\mathrm{SBS}_{7}$.

However, this MRS method does not take into account the primary working paths, so we introduce another method to minimize rSBSs with groups (MRG).

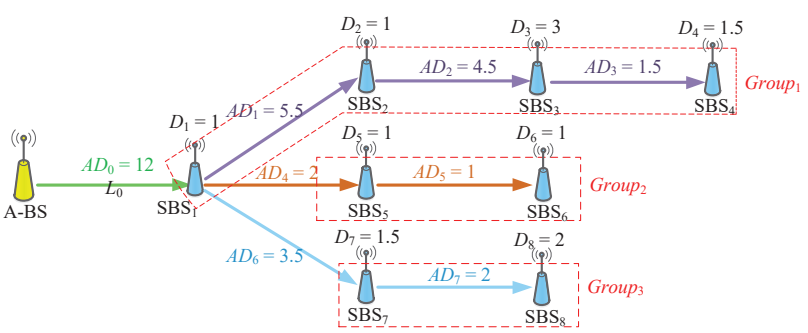

Figure 4: An example for minimizing rSBSs with groups.

In the MRG method, if the current capacity of link $\mathcal{L}$ drops below $A D_{\mathcal{L}}$, we first group all SBSs backhauled by link $\mathcal{L}$ according to their primary paths in set $G$. As an example, in Fig. 4, when $C_{\mathcal{L}_{0}}<A D_{0}$, all backhauled SBSs can be divided into three groups based on their primary paths. Then we start to minimize the number of groups that cannot be fully satisfied, i.e., maximize the number of satisfiable groups by solving the similar knapsack-like problem:

$$
\begin{gathered}
\max _{G} \sum_{g \in G_{l}} g_{i} \\
\text { s.t. } \sum_{i \in G_{l}} A D_{g_{i}} \cdot g_{i} \leq C_{\mathcal{L}}, g_{i} \in\{0,1\}
\end{gathered}
$$

where each $g_{i}$ includes the grouped SBSs backhauled by the logical link $\mathcal{L}$, and $A D_{g_{i}}$ is the aggregated data-rate demand of each group. After obtaining the groups that can be fully satisfied, the remaining items in $G$ are the groups $g_{r}$ that need to be reconfigured. Starting from the $g_{r}$ that comprises the maximum number of SBSs, we break $g_{r}$ and repeat solving the problem in Eq. (2)-(3) with the remaining $C_{\mathcal{L}}$, so the minimum number of rSBSs in each group is obtained.

In this way, for each group $g_{r}$ that is not fully satisfied, we only need to reconfigure the new backhaul path for the rSBS closest to the degraded link $\mathcal{L}$, i.e., the leader rSBS (L-SBS), because the paths from the L-SBS to other non-leader rSBSs (NL-SBS) can still be maintained within the group. Different from the MRS method that will alternate new backhaul paths for all rSBSs, here we try to force rSBSs in one group and then only take care of the leader rSBS, which can reduce the reconfiguration overhead.

Note that for those NL-SBSs in each group $g_{r}$, we also need to check whether or not each of them violates following constraints:

- Hop-count constraint (HC): The total hop counts of the new reconfigured path and the maintained path for the NL-SBS should be less than the hop-count threshold $H$.

- Data-demand constraint (DC): The available capacity of that new reconfigured path should be able to satisfy the data demand of the non-leader SBS.

If either $H C$ or $D C$ is violated, we need to select a backup path for that NL-SBS from its own SS. Note that the NL-SBS that has fewer backup paths should be checked first, because it has weaker network-level blockage tolerance and should be taken care of in the network first.

Here, the MRG method is adopted as an example shown in the Algorithm 2. As the procedure mentioned above, the reconfigured groups $g_{r}$ and corresponding rSBSs can be obtained by solving a knapsack-like problem (Lines 6-14). Then, the network-level reconfiguration is activated to select a new network path for the 
leader SBS in $g_{r}$, and we always select the backup path that has the maximum achievable throughput from its SS (Line 16). Here the achievable throughput performance $(T h)$ of each backup path $B P_{i}$ can be evaluated as follow:

$$
T h_{i}=\min _{\mathcal{L}_{j} \in B P_{i}}\left\{C_{\mathcal{L}_{j}}-A D_{\mathcal{L}_{j}}\right\}
$$

which is determined by the extra available capacity of the bottleneck logical link $\mathcal{L}_{j}$ within this path. Note that if no backup paths can meet its own data-rate demand, we have to give up selecting new paths for this SBS. For those NL-SBSs, if either $H C$ or $D C$ is violated, a new path will be selected in the same way (Lines 18-22). In the end, the aggregated data-rate demand set $A D$ and the backhaul path set NP need to be updated (Line 23).

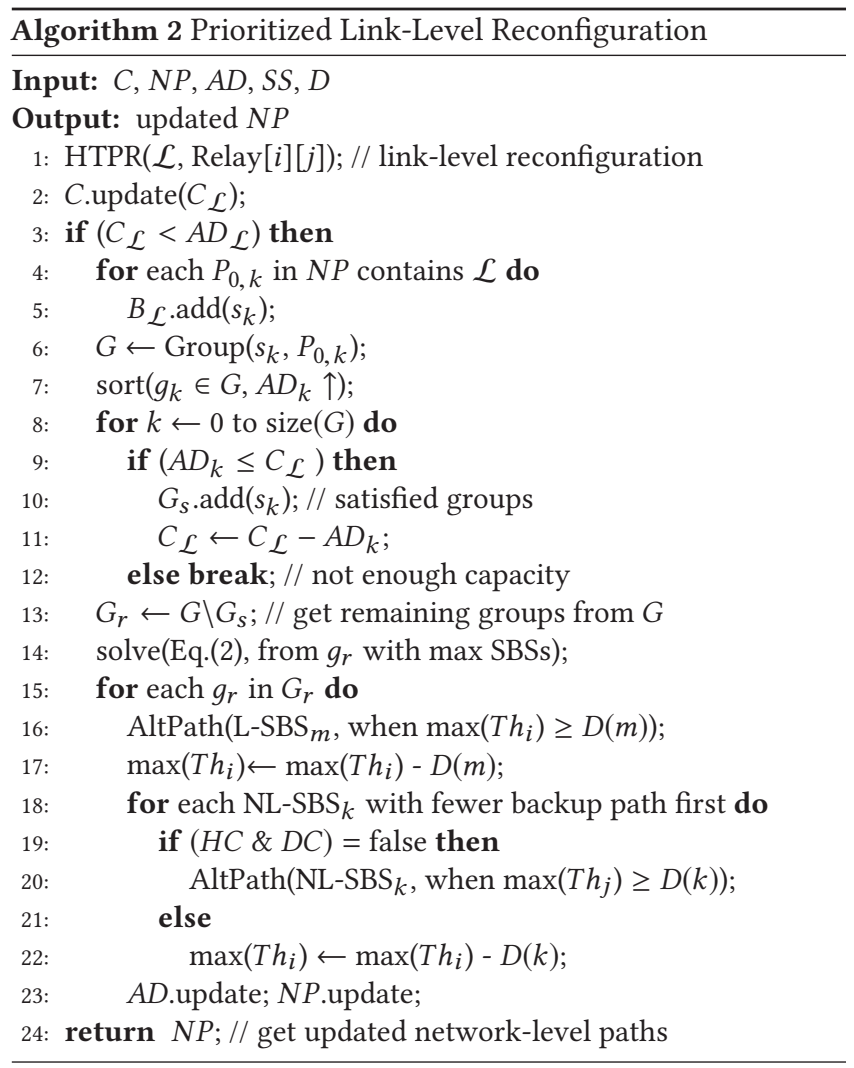

\subsection{High-Throughput Multi-path Reconfiguration}

To further improve throughput performance with blockages and better leverage the cooperation between the A-BS and SBSs, we introduce another reconfiguration approach based on the previous PLLR algorithm, which is referred to as high-throughput multipath reconfiguration (HTMR) scheme. This approach is trying to use the primary path and backup paths in combination for data transmission when the blockage occurs, which will work well in networks that support multi-path routing. As a primary relay path's performance is degraded due to link-level reconfiguration around obstacles, some of its traffic can be off-loaded to backup paths so that the reconfigured relay path and several alternative routes are used at the same time. Before adopting this algorithm instead of PLLR, we should pre-evaluate how much performance increase can be achieved compared to the PLLR scheme, since the benefit will depend on the overall network topology and the traffic flows, and HTMR scheme should be used for path recovery in the scenario where link outages occur infrequently and it can provide substantial benefits on throughput, and is therefore worth the added network control complexity for multi-path transmissions.

First, we need to solve a network flow problem in order to determine how to split traffic among the degraded primary path and backup paths which are totally disjoint. To split the traffic among multiple available paths, assuming there are $n$ possible network paths between A-BS and $\mathrm{SBS}_{i}$ with the data demand $D$, their respective achievable data rates $R_{i}$ can be obtained according to Eq. (6). In this way, the required transmission time over each path is $t_{i}=d_{i} / R_{i}\left(i \in P_{S}\right)$, where $d_{i}$ is the traffic assigned on path $i$. Assuming the data $d_{i}$ over each path is transmitted from A-BS to $\mathrm{SBS}_{i}$ at the same time, we can get the total required time $T_{r}$ until all data $D$ has been received as follow:

$$
T_{r}=\max \left\{\frac{d_{1}}{R_{1}}, \frac{d_{2}}{R_{2}}, \ldots, \frac{d_{n}}{R_{n}}\right\}
$$

Thus the end-to-end throughput can be obtained as $D / T_{r}$. To maximize the throughput performance by splitting traffic over multiple paths, we formulate the optimization problem as follows:

$$
\begin{aligned}
& \max _{\left\{d_{i}\right\}_{i \in P_{s}}} D \cdot \min \left\{\frac{R_{1}}{d_{1}}, \frac{R_{2}}{d_{2}}, \ldots, \frac{R_{n}}{d_{n}}\right\} \\
& \text { s.t. } \sum_{i \in P_{s}} d_{i}=D, R_{i} \geq 0, d_{i} \geq 0 .
\end{aligned}
$$

Theorem 1. To maximize the end-to-end throughput with given data demand D between A-BS and SBSs along $n$ routes, the assigned traffic for each route is proportional to its achievable rate, i.e.,

$$
d_{i}=\frac{D \cdot R_{i}}{\sum_{i \in P_{s}} R_{i}}(1 \leq i \leq n) .
$$

Proof. The objective function in Eq. (8) can be rewritten as $\max D \tau, R_{i} / d_{i} \leq \tau \forall i$. By making each $R_{i} / d_{i}$ be equal, the KarushKuhn-Tucker condition will be satisfied and we can get the optimal solution as $\sum_{i \in P_{s}} R_{i}$. Therefore, the optimal value $d_{i}$ is obtained as $D R_{i} / \sum_{i \in P_{s}} R_{i}$, which is proportional to $R_{i}$. If each $R_{i} / d_{i}$ is not split equally, which means that $d_{j}=\left(D \cdot R_{j} / \sum_{i \in P_{s}} R_{i}\right)-\epsilon$, and this would result in another split data $d_{k}=\left(D \cdot R_{i} / \sum_{i \in P_{s}} R_{i}\right)+\epsilon$ since the total demand should be unchanged. Therefore, $R_{k} / d_{k}$ becomes the bottleneck value and will reduce the original optimal solution.

As an extension of the PLLR scheme, the basic logic of HTMR algorithm is similar to Algorithm 2, but the main difference is the method to select network-level paths for each rSBS (L-SBS or NLSBS), which is shown in Algorithm 3. First, if the degraded logical link $\mathcal{L}$ 's capacity is not used up (Line 2 ), both the primary path $P_{0, k}$ and backup paths can be possibly used for rSBS, otherwise only backup paths will be used for reconfiguration. In both of these cases, we first need to find the disjoint backup paths in SS (Line 4). Here, an approach referred to as joint-weight selection is designed 
to obtain the maximum number of disjoint backup paths in SS (shown in Eq. 10), where each selected path $p_{i}$ does not have any overlapping logical links $\mathcal{L}$ with other paths.

$$
\begin{gathered}
\max _{P} \sum_{i \in S S} p_{i} \\
\text { s.t. } \mathcal{L}_{p_{i}} \cap \mathcal{L}_{p_{j}}=\emptyset, i \neq j, p_{i, j}=\{0,1\}
\end{gathered}
$$

In the case where the primary path $P_{0, k}$ can still be used, we need to put $P_{0, k}$ in SS since it can also be viewed as one of backup paths (Line 3). Then, we start to find disjoint paths in $S S_{k}$ (Lines 14-22). First, the joint weight $W$ for each backup path $B P_{i}$ is calculated, which represents the number of paths overlapping with $B P_{i}$ in $S S_{k}$, and these joint paths are recorded in $P$ (Lines 17-19). After that, we select the backup path $B P_{m}$ with minimum weight $W$ and put it into $M P$ (Lines 20-21). Note that if there exist multiple paths with the same minimum $W$, the one which has higher throughput will be selected. Finally, we obtain the maximum number of disjoint backup paths of $P_{0, k}$ until $S S_{k}$ is empty (Line 15). When the primary path cannot be used (i.e., $C_{\mathcal{L}}=0$ ), we can find maximum number of disjoint backup paths in $S S_{k}$ without $P_{0, k}$ in the same way.

After obtaining the multi-path set $M P$, the available backup paths are sorted in order of decreasing rate (Line 5), and the paths with highest rates will be iteratively selected until the data demand $D_{k}$ at $\mathrm{rSBS}_{k}$ is satisfied (Lines 7-10). In the end, we split the data demand $D_{k}$ according to Theorem 1 , and assign the traffic to each selected path in $M P^{\prime}$ (Line 12).

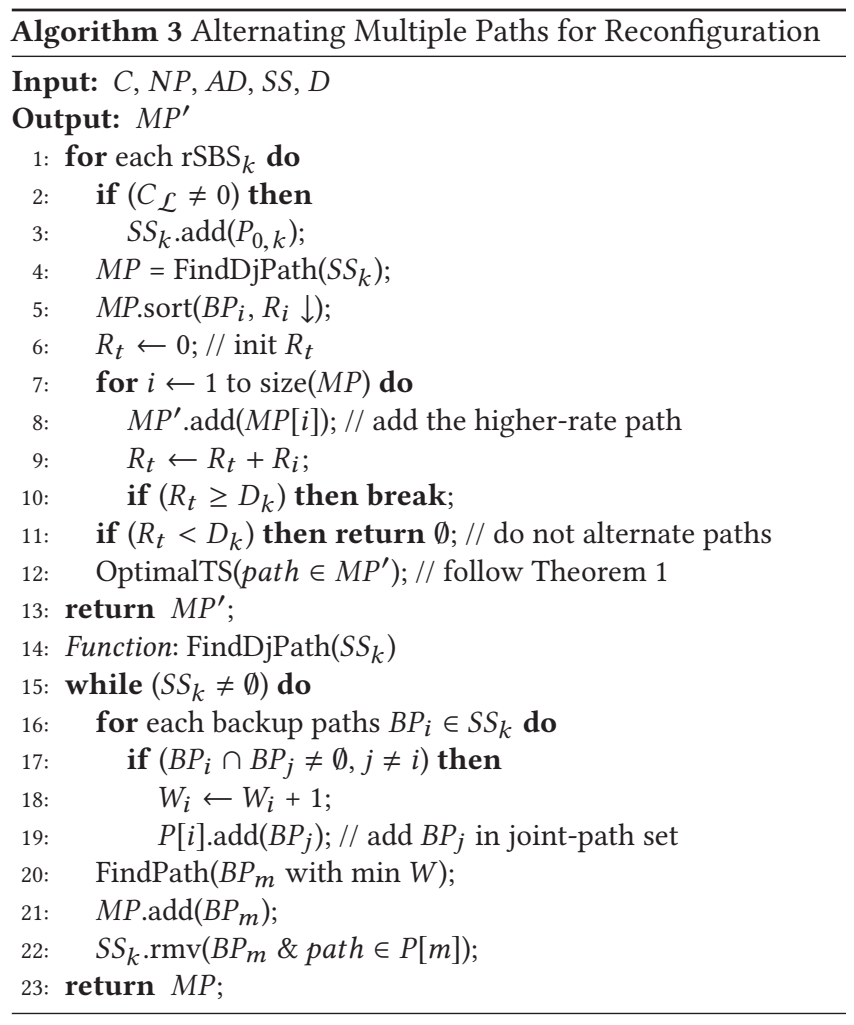

\section{NUMERICAL SIMULATIONS AND RESULTS}

In the face of obstacles that necessitate path recovery in mmWave backhaul networks, we evaluate the network performance including the throughput and blockage tolerance with different reconfiguration schemes. Here we conduct simulations where the mmWave backhaul network is deployed based on the Manhattan urban deployment shown in Fig. 1. With a single centralized A-BS, 19 SBSs at street corners and a number of relays along the roadside are selected. In this scenario, the established logical links between each pair of BSs, which consists of several physical links in a triangularwave topology, form a mesh-like network that covers the area of over $1.2 \mathrm{~km}^{2}$.

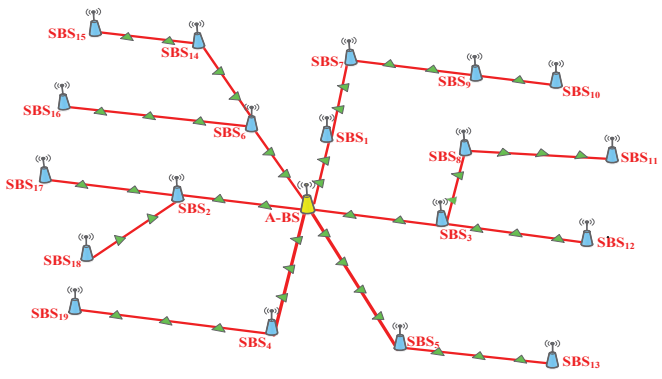

Figure 5: Primary backhaul paths in the mmWave backhaul network model.

In Fig. 5, without any link failures, we can compute that the network can support around 3 Gbps data demand for every SBS since each logical link (relay path) can achieve a throughput of around $13 \mathrm{Gbps}$. Considering potential blockages, we investigate the backhaul network survivability (BNS) [17] and satisfiable data demand for each SBS with our proposed reconfiguration schemes.

All evaluations are done at the mmWave frequency of $60 \mathrm{GHz}$ with a $2.16 \mathrm{GHz}$ bandwidth. The directional antenna gains $G_{t}, G_{r}$ of each wireless node are $23.18 \mathrm{dBi}$ and the transmit power is 1 watt. The attenuation from oxygen absorption is $17 \mathrm{~dB} / \mathrm{km}$, and a $15 \mathrm{~dB}$ link margin that covers rain attenuation and noise margin is included. Obstacles are generated randomly in the streets and modeled as rectangular vehicles, where their centers fall within the road and form a homogeneous Poisson point process (PPP) of density $\lambda$, the widths and lengths are assumed to be i.i.d. distributed and follow the normal distribution $\mathcal{N}\left(\mu_{w}=2.3 \mathrm{~m}, \sigma_{w}=0.8\right)$ and $\mathcal{N}\left(\mu_{l}=8.0 \mathrm{~m}\right.$, $\sigma_{l}=2.5$ ), and orientations are the same as the road's direction.

\subsection{Network and Path Survivability}

First, we evaluate the performance of the proposed PLLR scheme based on minimize rSBS method (MRS) and minimize rSBS with groups method (MRG), respectively. The aggregated user demand of each SBS is assumed to be around $1 \mathrm{Gbps}$, which follows the normal distribution $\mathcal{N}\left(D_{\mu}=1, D_{\sigma}=0.3\right)$. On hundreds of simulation runs with random-obstacle scenarios, Fig. 6 shows that MRS and MRG provide very similar BNS across a range of obstacle densities and the achieved BNS is relatively high (above $85 \%$ for all but the highest density). However, by adopting MRG method in reconfiguration schemes, the same high BNS can be achieved with fewer reconfigured network-level paths (see Fig. 7), especially when the obstacle density is not very high. This is because most of time we only reconfigure a new path for the leader rSBS instead of all rSBSs in the group, which substantially reduces the reconfiguration overhead. Based on this comparison, we adopt the MRG method in both PLLR and HTMR schemes in the remainder of the results. 


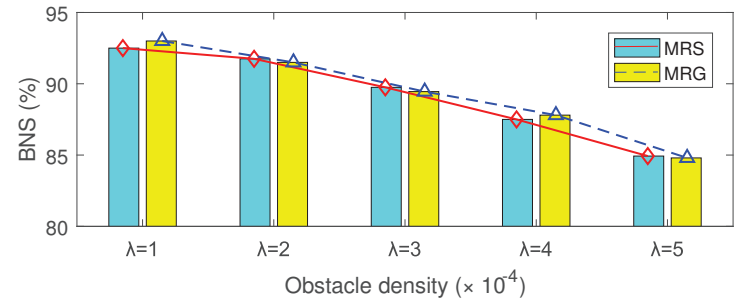

Figure 6: Survivability comparison between MRS and MRG.

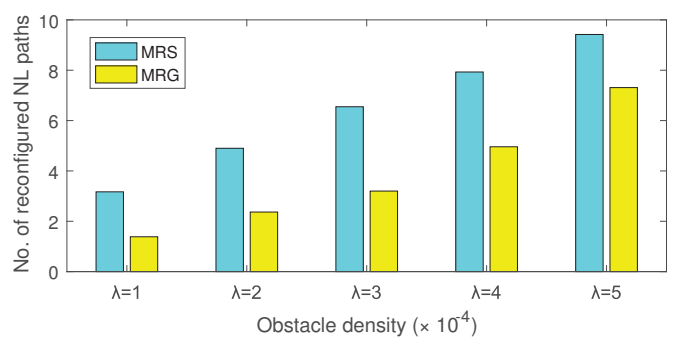

Figure 7: Number of reconfigured paths comparisons.

Second, we show the survivability performance with different reconfiguration schemes. As a comparison, an upper bound of BNS is also reported by solving the feasible-flow problem. By simulating a large number of random-obstacle cases, Fig. 8(a) shows the survivability performance of different reconfiguration schemes and the upper bound (labeled OPT). It is observed that the proposed PLLR and HTMR schemes provide significantly better robustness than purely link-level and purely network-level reconfiguration approaches, where BNS is obviously improved and still over $80 \%$ even with a high density of obstacles. Of particular note, the NLR scheme that is adopted by most previous work has worse performance than LLR, and has poor survivability with multiple blockages. On the other hand, we can see that the BNS of our proposed schemes is very close to the upper bound on BNS, and as the obstacle density increases, the performance gaps between the two proposed schemes and the upper bound BNS are 1.2\% 4.8\% and 3.5\% 8.4\%, respectively. Since the PLLR scheme only supports single-path routing and always reconfigures one path for each source-destination pair, the performance gap of PLLR scheme is a little larger than that of HTMR scheme, but it is still around 5\% with a not very high obstacle density.

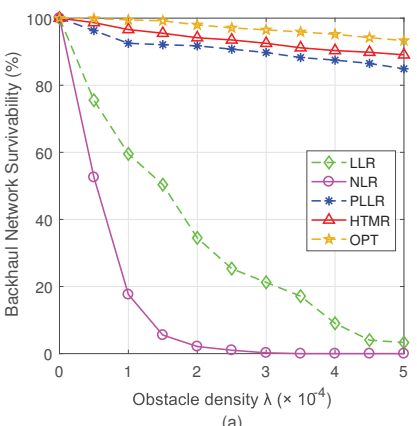

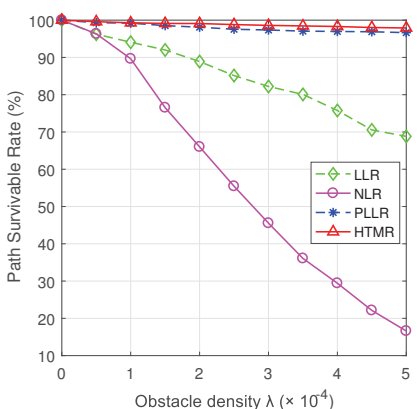

(b)
Figure 8: Network and path survivability comparisons.
Fig. 9 shows how the BNS of the proposed schemes and the upper bound vary with increasing data demand, for an obstacle density of $\lambda=2 \cdot 10^{-4}$. As the demand increases, pure network level and pure link level reconfiguration fail to provide survivability in almost all cases. However, our proposed schemes maintain fairly high BNS and remain close to the upper bound over all simulated demands.

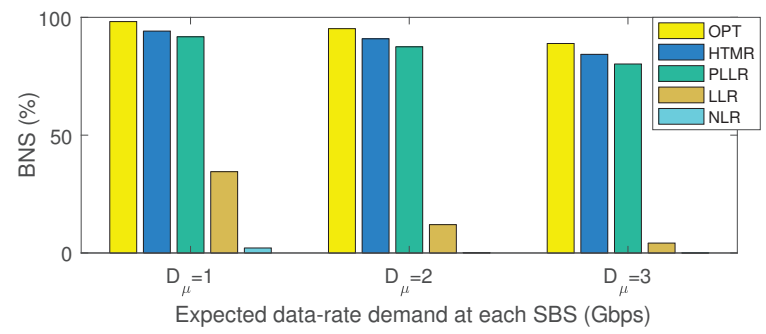

Figure 9: Network survivability comparisons with different expected data-rate demands.

To get more fine-grained results, we also evaluate the path survivable rate (PSR), which is defined as the fraction of total backhaul paths that remain connected with reconfiguration after blockages occur. Fig. 8(b) shows that PLLR and HTMR can provide over $97 \%$ PSR as the number of obstacles increases, which means that the communication from the A-BS to each SBS would be largely unaffected, even with multiple obstacles. Thus, with the proposed schemes, the blockage tolerance of the entire backhaul network is highly enhanced.

\subsection{Throughput Performance}

In this part, we evaluate whether or not the proposed reconfiguration algorithms can maintain high throughput with blockages, and the satisfied-BS percentage (SBP) is evaluated. SBP indicates the fraction of SBSs that have their respective data demands satisfied. Here, the aggregated user demand of each SBS is assumed to follow the normal distribution $\mathcal{N}\left(D_{\mu}=2, D_{\sigma}=0.3\right)$. From Fig. 10, we can see that HTMR scheme has the best SBP compared with other schemes, since it splits traffic for transmission on both the primary working path and backup paths, which compensates for degraded throughputs on the reconfigured relay paths.

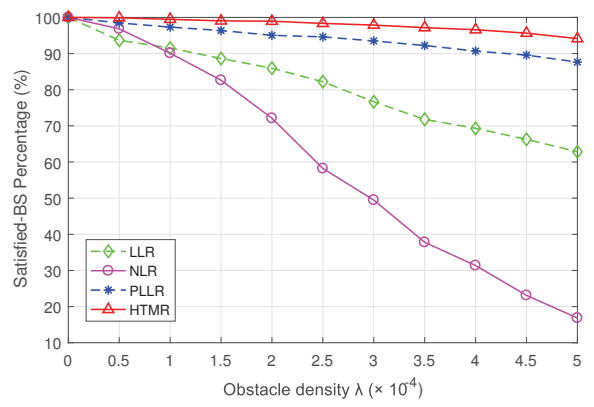

Figure 10: SBP comparisons among different schemes.

In addition, we vary the data demand $D_{\mu}$ of each SBS, and it is obvious to see that the SBP will decrease as $D_{\mu}$ increases with both PLLR and HTMR schemes (shown in Fig. 11 (a) and (b), respectively). By comparing the SBP based on the same $D_{\mu}$ between these two schemes, the HTMR scheme can satisfy more SBSs than the PLLR 
scheme with the same obstacle density, and the SBP is improved by more than $20 \%$ when $D_{\mu}=3$ and the obstacle density is high. Because of such substantial benefits on throughput performance, it might be worth adopting HTMR despite its added complexity.

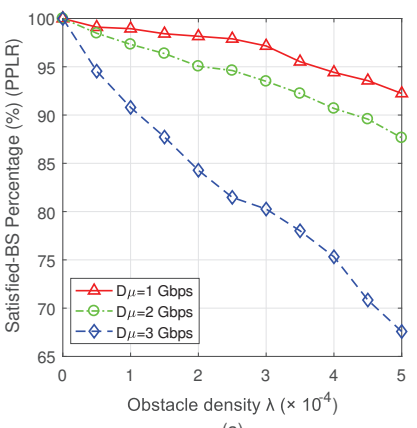

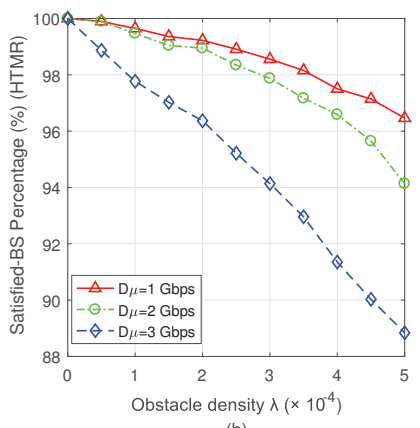

Figure 11: SBP for PLLR and HTMR with different $D_{\mu}$.

\subsection{Hop-constraint effects}

Our problem formulation assumes that each eligible backup path selected for a specific backhaul path should satisfy a hop-number constraint, i.e., the total hops (including dedicated relays and relayedSBSs) along this path must be fewer than a hop-number threshold $H$. Here we investigate how this $H$ affects network survivability. With the obstacle density $\lambda=1.2 \cdot 10^{-4}$ and data demand at each SBS of around $1 \mathrm{Gbps}$, Fig. 12 shows that the BNS will increase as $H$ increases for all reconfiguration approaches except the LLR scheme. This is because with larger $H$, more backup paths for each backhaul path are put in the SS, which improves the network-level reconfiguration, but this has no effect on a purely link-level reconfiguration approach. In addition, by increasing $H$, PLLR and HTMR can even provide nearly $100 \%$ BNS, and NLR can also possibly provide better blockage tolerance than LLR. However, a larger $H$ results in higher end-to-end latency and, therefore, this trade-off between latency and robustness in the network would need to be carefully considered with respect to application latency requirements.

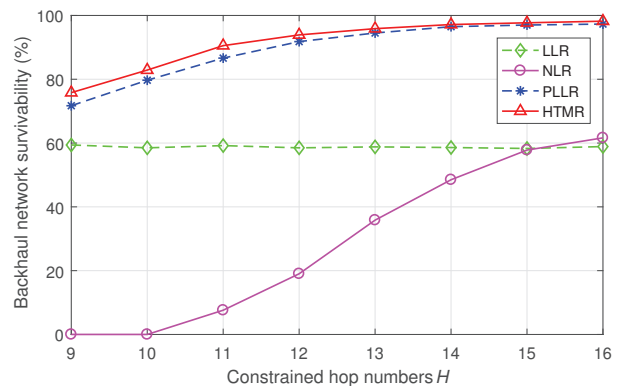

Figure 12: BNS comparisons with different schemes vs. constrained hop number $H$.

\section{CONCLUSION}

In this paper, we considered the problem of survivability in mmWave backhaul networks, where a single A-BS serves as a gateway for a number of SBSs and relays deployed along urban streets. In such a scenario, joint link-level and network-level reconfiguration schemes are proposed to overcome blockage and failure effects. These schemes combine relay path reconfiguration with alternate backup paths for fast path recovery. Through simulation results, the performance of proposed schemes is shown to be always superior to purely link-level/network-level reconfiguration schemes. The approaches can provide a substantial improvement in backhaul network survivability, and also maintain backhaul throughputs, achieving the Gbps data demand for each SBS even in the presence of multiple blockages/failures. Future research will focus on path recovery in other network topologies of urban environments.

\section{ACKNOWLEDGMENTS}

This research was supported in part by the National Science Foundation through Award CNS-1813242.

\section{REFERENCES}

[1] F. Wei, Y. Li, D. Jin, L. Su, and S. Chen, "Millimetre-wave backhaul for 5G networks: Challenges and solutions"[J]. Sensors, 2016, 16(6): 892.

[2] M. Kulkarni, A. Ghosh and J. Andrews, "How Many Hops Can Self-Backhauled Millimeter Wave Cellular Networks Support?”. arXiv:1805.01040, 2018.

[3] Q. Hu and D. Blough,"Relay Selection and Scheduling for Millimeter Wave Backhaul in Urban Environments", IEEE MASS, 2017.

[4] M. Jaber, et al., "Wireless Backhaul: Performance Modeling and Impact on User Association for 5G" [J], IEEE Transactions on Wireless Communications, 2018.

[5] B. Sahoo, C. Yao, H. Wei, "Millimeter-wave multi-hop wireless backhauling for 5G cellular networks". IEEE Vehicular Technology Conference, 2017.

[6] T. Bai, R. Heath, "Coverage and rate analysis for millimeter-wave cellular networks", IEEE Transactions on Wireless Comm., 2015.

[7] Y. Liu and D. Blough, "Analysis of Blockage Effects on Roadside Relay-assisted mmWave Backhaul Networks", IEEE ICC, 2019.

[8] S. Sur, V. Venkateswaran, X. Zhang, et al., "60 ghz indoor networking through flexible beams: a link-level profiling", in ACM SIGMETRICS. ACM, 2015.

[9] X. Ge, H. Cheng, M. Guizani and T. Han, "5G wireless backhaul networks: challenges and research advances”. IEEE Network, 2014.

[10] R. Rony, et al., "Joint access-backhaul perspective on mobility management in 5G networks", IEEE Conf. on Standards for Comm. and Networking (CSCN), 2017.

[11] Q. Hu and D. Blough, "Optimizing Millimeter-Wave Backhaul Networks in Roadside Environments," IEEE International Communication Conference (ICC), 2018.

[12] S. Ramamurthy, L. Sahasrabuddhe, B. Mukherjee, "Survivable WDM mesh networks". Journal of Lightwave Technology 21.4 (2003): 870.

[13] D. Xu, Y. Chen, Y. Xiong, C. Qiao, X. He, "On finding disjoint paths in single and dual link cost networks". IEEE INFOCOM 2004.

[14] S. Paira, M. Chatterjee, S. Khanam, et al., "GSWA: A Survivable Dynamic Multicast Efficient RWA Scheme in WDM Mesh Networks", IEEE ICCCNT, 2018.

[15] M. Elappila and S. Chinara. "Dynamic Survivable Path Routing for Fast Changing IoT Network Topologies”. IEEE TrustCom/BigDataSE, 2018.

[16] A. Bigdeli, A. Tizghadam and A. Leon-Garcia, "Survivable routing using path criticality", IEEE ICNC 2012: 793-797.

[17] M. Parandehgheibi, H. Lee, et al., "Survivable path sets: A new approach to survivability in multilayer networks"[J]. Journal of Lightwave Technology, 2014.

[18] A. Jabbar, et al. "Survivable millimeter-wave mesh networks." Computer Communications 34.16 (2011): 1942-1955.

[19] Y. Niu, et al.. "Exploiting multi-hop relaying to overcome blockage in directional mmWave small cell", fournal of Communications and Networks, IEEE, 2016.

[20] S. Biswas, S. Vuppala, J. Xue, and T. Ratnarajah,"On the performance of relay aided millimeter wave networks”, IEEE 7. Sel. Topics Signal Process., 2016.

[21] Y. Liu, Q. Hu, and D. Blough, "Blockage Avoidance in Relay Paths for Roadside mmWave Backhaul Networks”, IEEE PIMRC, 2018.

[22] A. Nasr and Y. Fahmy, "Millimeter-wave wireless backhauling for 5G small cells: Scalability of mesh over star topologies", IEEE WoWMoM, 2017.

[23] Y. Liang, B. Li and M. Yang, et al., "MAC protocol framework for 5G mmWave backhaul network", IEEE ICSPCC, 2016: 1-6.

[24] R. Taori and A. Sridharan, "Point-to-multipoint in-band mmwave backhaul for 5G networks[J]", IEEE Communications Magazine, 2015, 53(1): 195-201.

[25] J. Du, E. Onaran, et al., "Gbps user rates using mmWave relayed backhaul with high gain antennas", IEEE fournal on Selected Areas in Comm., 2017.

[26] B. Xie, Z. Zhang, and R. Hu, "Performance study on relay-assisted millimeter wave cellular networks", IEEE Proc. Vehicular Technology Conference, pp. 1-5, 2016.

[27] Y. Liu, Q. Hu and D. Blough, "Technical Report for Blockage Type Detection Process". (available at: http://blough.ece.gatech.edu/BTDTechReport.pdf) 